\title{
Clinical aspects of the electrocardiogram
}

\author{
Fred Fricke \\ University of Nebraska Medical Center
}

This manuscript is historical in nature and may not reflect current medical research and practice. Search PubMed for current research.

Follow this and additional works at: https://digitalcommons.unmc.edu/mdtheses

Part of the Medical Education Commons

\section{Recommended Citation}

Fricke, Fred, "Clinical aspects of the electrocardiogram" (1931). MD Theses. 151.

https://digitalcommons.unmc.edu/mdtheses/151

This Thesis is brought to you for free and open access by the Special Collections at DigitalCommons@UNMC. It has been accepted for inclusion in MD Theses by an authorized administrator of DigitalCommons@UNMC. For more information, please contact digitalcommons@unmc.edu. 
OLINICAL ASPECTS OF THE EIECTROOADDIOGRA

\section{FRED FRICKE}


CLINIOAI ASPEOTS OF THE ELECTROCARDIOGRAN

The electrocardiograph is an instrument of comparatively recent invention for use in the study of heart cases. Its purpose is to photograph the changes in electrical potential which take place in the heart during activity. It was known as early as 1856 that an electric current was produced by the contracting heart muscle (I), and in I885 KoIliker and fuller (2) showed the presence of action currents in heart muscle by laying the nerve of a nerve-muscle preparation across a contracting heart, and observing the activity of the nerve muscle preparation. Two years later waller demonstrated by means of a mercury galvenometer that the current produced by the active heart muscle couId be lead from the surface of the body if a satisfactorily sensitive instrument was available to record it. Einthoven, of Amsterdam, made the recording of these heart currents possible by the invention of the exceedingly sensitive string galvanometer, which was first described by him in 1903. Sir Thomas Lewis (3) was the first person to use the electrocardiograph for clinical purposes, and published the results of his studies in 1906 and 1908. The use of the instrument was then rapidly taken up abroad, and was introduced in this country between 1910 and 1913. 
The electrocardiograph (4) which employs Einthoven's string galvanometer consists of an electromagnet activated by a storage battery of five or six amperes which gives about ten volts. The "string" which is suspended between the poles of this electromagnet consists of a filament of platinum or silvered quartz about half as thick as the diameter of a red blood cell or two to four microns. When the current from the patient's heart passes along this string at right angles to the lines of force of the electromagnetic field the string will be deflected to one side or the other depending upon the direction of the current which is passing thru it. By means of an arc light passing thra a system of condensing lenses the shadow of the string is magnified, focused, and printed on a motor-driven plate or film. This photographic record of the heart action is known as an electrocardiogram. The string can be adjusted to a proper tension so that one centimeter deviation of the shadow will be caused by one millivolt of current. A single dry cell battery is used to counter-balance the sin in currents such as those set up by glandular activity. Vertical lines are photographed on the plate to determine the time. This is usual Iy done by means of a spoked wheel which revolves 
at a constant known rate. Horizontal lines are also printed on the film so that the height of the shadows can be determined. The electrodes used to connect the patient with the electrocardiograph may be German silver, zinc or Iead plates, or vessels filled with salt solution.

More recently the Victor electrocardiograph (5) has been piaced upon the market and is now used quite extensiveIy in place of the string galvanometer of Einthoven, it has less sensitive torsion galvanometer, consisting of a fixed U magnet with an enameled iron vane suspended by a phosphor-bronze Hibbon, with a mirror attached to the vane which reflects the beam of light on to the photographio plate. This part of the electrocardiograph is known as an oscillograph. To compensate for the lesser sensitivity of this instrument amplifying tubes are used which multiply the heart current about three thousand times.

When the body is at rest the heart is practically the only active muscle present, so any current that is produced must come from the heart muscle (6). Therefore, electrodes placed angwhere on the body with the heart between them will register the electrical activity of the heart. In oroer to 
make the work more uniform special parts of the body were used for attachment of the electrodes. These were the right and left arms which constitute lead $I$, the right arm and Ieft leg which lead II, and the left arm and left leg which is lead III. Three different leads are used because each one gives a different result, since the total current of the heart is in the same direction for each lead, but the receiving apparatus is in a different relation to this current. Therefore, under abnormal conditions one lead may show certain characteristics not present in either of the other leads. The action currents of the heart (I) which the electrocardiograph records are produced because the muscle fibers of the heart are not always all contracting at the same time, and the actively contracting muscle fibers acquire an electrical potential difference from that of the non-contracting fibers. Therefore, as long as there is a difference in activity of the heart muscle fibers there will be a difference in potential, and an electric current will be produced. However, if all of the muscle fibers are contracting at the same time they will all have the same potential and no current will be present. Since the electrocardiogram of 
a given heart is normally the same during each cycle it can be assumed that the contraction always starts in the same place and travels over the same route, producing the same difference of potential. From experimental work the normal starting point (7) for the heart muscle contraction has proven to be a mass of speriolized muscle tissue in the wall of the right auricle near the superior vena cava, known as the sinoauricular node or $\mathbf{S - A}$ node. From this point the impulse, which precedes and causes the contraction, passes thru the auricular muscle, the auriculo-ventricular or $A-V$ bundle or bundie of His, the right and left brances of this bundle, and finalig to the entire ventricular wall thra the fine terminations of these branches, which are known as the fibers or corpuscles of Purkinje The electrocardiogram of a regularly beating heart (8) whose impluses rise at and travel over the usual route described above will present certain characteristics to be looked for in all electrocardiograms. The record of each cyoie in any given lead for a single patient will appear exactly the same as every other cycle record in that lead. Hinor variations may always be expected in records from different leads in the same patient, or in the same lead from different patients. 
There are normally three upward curves to each heart cycle, and two very small downward curves of less amplitude and less constancy. The first upward curve is known as the P-wave, and represent the electrical disturbance associated with auricular systole. It is a rounded elevation varying in height from bare perceptibility to two millimeters. The second upward deflection is known as the R-wave, and consists of a sharply pointed or spiked deflection, from seven to sixteen millimeters, in height in the lead in which it is greatest. The $\mathrm{g}$-wave is usually highest in lead II, always being equal to the sum of the R-waves in leads II and III. Ih $Q$ and $S$ waves, if present, appear as sudden downward deflections of small amplitude just before and after the $\mathrm{R}$-wave respectively. No pathological significance is attached to the absence of either or both of these waves. The $Q_{2} B$, and $\mathrm{D}$ waves are usually spoken of as the $Q R S$ interval or complex, and represents the beginning of ventricular activity. The S-wave, after a period of inactivity, is followed by an upward deflection usuelly not over five millimeters in height, and with a comparatively rounded apex. This wave represents the end of ventricular systole, 
the difference of potential resulting from the cessation of activity of the muscle, which does not all cease contracting at the same time. The distance between the $S$ and waves where the line is straight, which is known as an isoelectric period, is probably due to the entire ventricle being in contraction and, therefore, no electrical reaction is produced. The period from the beginning of the $2, R$, $S$ interval to the end of the $T$-wave represents Ventricular systole. The isaelectric period following the p-wave to the next P-wave occurs while the heart is completely inactive or during diastole. A very slight upward deflection known as the U-wave is sometines seen during this period, but it is very inconstant and no significance is attached to its presence or absence. The period from the beginning of one P-wave to the beginning of the next P-wave represents a complete heart oycle. The space from the beginning of the P-wave to the begiming of the $2, \mathbb{R}, \mathrm{S}$ complex is known as the $P-R$ intervel, and nomally is not over 0.20 of a second in duration. The duration of the $Q, I, S$ interval is

5. also of considerable importance, 0.10 of a second being the upper limit of normal. In interpreting an electroca raiogram direction, amplitude, duration, and time relations of the various 
waves and intervals are important factors to note. The R-wave is related to the condition of the ventriculer musole, but often increases in amplitude after acute illnesses or with the over-activity of exophthalmic goiter, and decreases following prolonged severe physical exertion. The T-wave may be extra. large or occasionally inverted in lead III in the normal record (9), but is always upwardy directed in leads $I$ and II. Abnomal electrocardograms vary considerably in their appearance, and significance, depending upon the condition causing the abnormality. An arbitrary classification (7) of disorders in which the electrocardiograph is a useful aid will be given to simplify the matter of describing them. I Disorders of Rhythm. II Disorders of Bate. III Disorders of Conduction. IV Hyocardial Disturbances. V Congenital Abnomalities. One of the most common and simplest of the disorders of rhythm is sinus arrythmia (I). It is a normal change in the pulse rate, and the variations being synchronous with the respiratory phases due to vagus pressure. The pulse rate increases with inspiration and decreases during expiration. It is seen in practically all children and in many adults. The electrocaraiographic tracings are perfectly normal except for the regular veriation in the rete produced (page 9) 
by variations in the length of the diastole, or S-I interval. It is termed sinus arrythmia because the impuise arises in the simus or sino-auricular node. It is not a pathological condition, it produces no symptoms, and therefore, it requires no treatment.

The occurrence of extrasystoles (7) is also a common heart disorder, and is quite easily reoognized both clinically, and by means of the electrocardiogram. Extrasystoles may be divided into three groups, namely, Auricular, Ventricular, and Nodaz. An amricular consists of a premature impulse arising in the auricle and causing a complete heart beat. It is followed by a pause that is longer than normal, which is known as a compensatory pause. The electrocardiogram of this condition shows the T-P interval preceding the premature beat shorter than normal, and a prolonged T-P interval following the early contraction. The P-wave of the premature contraction may be normal, lost in the preceding $\mathbb{T}$-wave, exaggerated, flat, or inverted, depending upon the prematurity and the point of origin of the impulse within the auriale. The interval of the heart sycle preceding the auricular extrasystole plus the premature beat is slightly shorter than 
two normal beats.

In ventricular extrasystoles the premature impulse arises at some point within the ventricle itself. It is not influenced by the P-wave, nor is the normal auricular activity interferred with. The $\mathrm{P}$-wave may be found just before the extrasystole occurs, or may be lost in $0, B, S$ complex of the premature beat, but it is always just where it would be nomally if no extrasys tole had occurred. The ventricular complex, however, is distorted because the impulse arises below the nomal level. The $Q, R, S$ interval is increased in duration over 0,20 seconds, the largest deflection is increased in amplitude, and there is usually thickening or slurring of the Iimbs. The T-wave is exaggerated both as to time and amplitude, and it points in the direction opposite that of the greatest deflection of the $0, R, S$ Group. Since the entire $Q, R, S$, and $T$ complex is not increased in length the S-T interval is practically absent. The ventricle from which the impulse for the extrasys tole arises can usually be determined by the directions of the greatest deflections of the $Q, R, S$ groups in each of the three leads. If the impulse starts in the right ventricle the greatest deflection of the 
Q, $\mathrm{B}, \mathrm{S}$ group is downward in lead $\mathrm{I}$ and upward in leads. II. and III. If the impulse arises in the left ventricle the greatest deflection of the $0, R, S$ group is upward in lead I and downward in leads II and III.

Nodal extrasys toles are those arising in the auricular-ventricular node. Since this node is at the junction of the auricles and ventricles the P-wave and $Q, R, S$ group will occur practically simultaneously. The portion of the node from which the impulse arises determines the exact time relationship of the auricular and ventricular curves. If the impulse arises in the upper part of the node the auricular contraction will just precede the ventricular contraction. If the impulse arises near the middle of the node the auricles and ventricles will contract together and the P-wave will be lost in the $2, R$, 5 group. If the lower portion of the node gives rise to the impulse the ventricle will contract first and the P-wave will be found just after the $0, B, S$ complex. In any of these conditions of nodal extrasystoles the impulse arises above the ventricles. Therefore, the $\mathrm{a}, \mathrm{R}, \mathrm{S}$ complex is always normal except when distorted by a synchronous $P$ wave. The P-wave if found, is usually inverted or distorted in some way because 
the impulse comes from the lower instead of the upper part of the auricles. Infrequently the auricular-ventricular node replaces the sinus node completely as the point of origin for the cardiac irmpulses (10). This condition is known as nodal rhythm, and if persistent indicates destruction of the sinoauricular node and may, therefore, be considered as evidence of considerable myocardial damage. The electrocardiogram in this condition shows a slow regular pulse of 35 to 50 beats per minute with all of the cycles showing one of the three types of abnomality just described as nodal extrasystoles. Extrasys toles may occur in normal hearts as the result of some stimulus such as coffee, alcohol, drugs, etc. In these conditions they are usually infrequent and not associated with any other abnormalities. If they occur frequent$1 \mathrm{y}$, in an irreguiar manner, or associated with other abnormalities they are usually due to some organic heart disease, and treatment should be directed toward the removal of the underIying pathology (II).

A condition in which the electrocardiograph is a valuable aid in diagnosis is auricular flutter. In this condition the impulses arise at a greatly increased rate, 
varying from 230 to 350 per minute. Their origin is some point in the auricle near the superior vena cava, but outside the sino-auricular node, and they travel in a circular course about the vena cavae. The P-waves are usually diphasic, espectally in Iead II, because of the unusual course of the impulse. The ventricular complexes are normal because the impulses arise above the auricular-ventricular node. There is practically always some block associated with auricular flutter, the most frequent type being $2: 1$ black, producing a ventricular rate one-half that of the auriole. A $3: I$ blocik 4:I blook, etc., are occasionally seen also, and infrequently the ratio of the auricular beats to the ventricular beats vary, producing what has been termed impure flutter (I3). Very rarely each auricular impulse reaches the ventricle, and the ventricle beats as rapidly as the auricle. This excessively rapid ventricular rate warrants a poor prognosis since heart fallure is likely to follow this over-exertion. The etiology of auricular flutter (II) is not known, and associated cardiac pathology is not constant. The treatment, however, is often very successful, even if the condition has lasted for months, as not infrequentIy happens. Digitalis is the arug of choice, and at first it slows the ventricular rate, but soon produces 
auricular fibrilation. If the drugs is not discontinued the rate very often returns to nomal. auinidin may be used in the same alone or with digitalis. Auricular fibrillation (13) is a more frequent condition which resembles flutter slightly. The impulse arises in the auricular muscle and takes a circular course, but unlike flutter the course is not constant, and it is supposed that impulses al so pass off at a tangent to the circular path. The rate of these impulses al though irregular due to their varied courses vary from 350 to 500 or more per minute. These impulses occur so rapidly that the auricle does not have time to respond to each one and remains in a state of fibrillation. The impulses Which reach the ventricle are variable as to number, and markedIy irregular. The most characteristic feature of the electrocardiogram of the fibrillating patient are the irregularity of the ventricular complexes and the absence of normal P-waves. The ventricular complezes may be normal or the B-waves nay vary in height from cycle to oycle. The auricular activity may be manifest on the electrocardiogram by fine twitohings seen during ventricular diastole and know as fibrillary or P-maves. 
Aurioular fibrillation is generally found with organic heart disease, especially rheumatic or mitral disease and toxic goiter. Whe prognosis of this condition with mitral disease is not favorable as a rule, al though if there is little other evidenoe of mascle damage the patient may Iive for some time if properly treated. Auricular fibrillation associated with thyroid disease is less serious, but its presence inoreases the seriousness of the thyroid condition(12). The habits of the patient (II) must be regulated, and digitalis is the chief drug to lessen the rate of the over-marked ventricle. It does this by impeding the passage of the impulse from the auricle to the ventricle, or increasing the heart blook. Guinidin is also very popular in the treatment of this condition, and often gives excellent results.

Disturbances of rate can be very accurately diagnosed by means of the electrocardiogram (14). If a bradycardia. or tachycardia is present the electrocardiograph will determine whether it is due to a disturbed conduction mechanism within the heart itself, or to some external cause. In the first instance some abnormalities in one or another of the waves will bo present, while in the latter case the cycles will be 
normal but either farther apart or closer together than usual. The most important pathological disturbance of the heart rate is the condition kmown as paraxysmal tachycardia (7). It consists of a sudden increase in rate, and a sudden return to normal, the attack lasting from a few seconds to several days or even two weeks. The shorter intervals are more common, a few hours being the most frequent length. The. impulses producing this condition are ectopic in origin, that is, they do not arise at the sino-auricular node. Auricular ectopic impulses are most frequentIy responsible for the paroxysms, but they may also arise in the auriculo-ventricular node or in the ventricle. Since each beat during the attack is an extrasystole the electrocardiographic curves will similate those described earlier for extrasystoles. The rapid rate will naturaliy place the heart cycles very close together, and the origin of the impulse may be hard to determine unless the beginning or the end of an attack is included in the record. The etiology of this condition is very indefinite. In one series of cases (II) approximately $22 \%$ were associated with mitral stenosis, $22 \%$ with symptoms of myocardial degeneration, $8 \%$ wh arterial disease including angina pectoris, 
$6 \%$ with renal disease and cardiac enlargement, 2 with thoracic anewrism, $2 \%$ wi th early pulmonary tuberculosis; and $38 \%$ without any other symptomatology. The symptoms associated with paroxysmal tachyoardia vary consfderably depending upon the length of the attack and the heart rate during the paroxysm. If the attack is of very short duration the patient may not be aware of it at all, whereas, in severe attacks complete incapacitation may be experienced due to precardial pain, palpitation, exhaustIon, coldness, sweating and gastrointestinal symptoms. The prognosis of this condition, while it shoula be guarded, is not very grave. Death has been reported due to congestive heart failure during an attack, but the great majority of cases recover. Complete freedom from recurrent attacks cannot be promised, although in young patients the prospects for it are fair. No treatment has yet been found. which constantly gives good results, although a great many things have been tried. The electrocardiogram is very helpful in the classification and diagnosis of the disorders of conduction, or heart block. 庄 rather infrequent type of this condition is known as sino-aurioular black or dropped beats (I4). The impulse arising at the sino-auricular fails to get to the auricular muscle or fails to arise at all. Therefore, the 
mricular muscle does not contract nor does the ventricle contract. Therefore, there are no curves on the electrocardiogram for a short period. The interval between the two Pwaves which includes the isoelectric period is slightly less than two normal cycles. The etiology of dropped beats is unknown, al though they occur more frecuently when the heart rate is rapid. They produce no symptoms and no treatment is required. A clinical diagnosis of dropped beats is frequently made from the compensatory pause following extrasystoles. A much more common conduction disorder is auriculoventricular heart block $(7)$. It consists of some interference with conduction at the auriculo-ventricular node or bundle of His, and may be divided into incomplete and complete block. The incomplete or partial block may be only severe enough to slow the impulse, which is demonstrated on the electrocardiogram by a prolonged $P-R$ interval of more than 0.20 seconds. If the condition is slightly orse an ocasional impulse may fail to reach the ventricle, and the $Q, R, S, T$ complex will be absent after the P-wave. Hore commonly the failure of the impulse to reach the ventricle occurs regularly. The ratio of impulses present (auricular contractions) to those which 
reach the ventricle may be $2: I, 3: 1,4: I$, etc., the condition then being termed 2:I blook, 3:I block, etc. These more severe types of insomplete block are usually associated with auricular flutter. The lesser incomplete block may result from orngs suoh as digitalis and morphin, or from the toxic condition accompanying such aiseases as pneumonia, diphtheria, and rheumatism. The cardiac condition itself is not harmful, and treatment should be directed toward the removal of the cause.

Complete heart black is a condition in which there is complete blooking of the passace of impulses from auricle to ventricle. An independent impulse then arises within the ventricle itself, the rate of these impulses usualiy being slower than that of the suricle, and entirely independent of it. The ventricular rate may be below fifty with an aurioular rate of over one hundred. The picture of complete bIock presented by the electrocardiogram consists of normal regularly recurring P-waves and a smaller number of normal, regular recurring $, A, 3, I$ corplexes, with no definite time relations between the two. Complete heart black (13) may be the result of more advanced toxic conditions 
similar to those causing partial heart-block, namely, drugs or certain infectious aiseases. Amoung the organic lesions which might be responsible for complete heart block are atherosclerosis, gumma, calcified areas, infarcts, and coronary disease. The symptoms and treatment will depend tapon the etiological factor.

A blook of either one of the main branches of the bundle of His will produce a quite cheracteristic electrocardiographic tracing. The P-wave will be normal, but there will. be a prolonged $A, R$, $S$ interval of more than 0.10 seconds, there will be notching or slurring (thickening of the limbs) of the major deflections of the $, R, S$ group, and a $T$-wave directed opposite to the largest deflection of the $2, B, s$ group. If the block is complete tilese abnormalities will be marked in all three Ieads. If the block is only a portion, or appears farther from the bundle of His on one of the small branches (arborization block) the finding on the electrocardiogram will not be as extensive. The ventriale in which the block occurs can usually be determined by the directions of the largest deflections of the $Q, B, S$ complex. In rightsided block the largest deflection will be downard in lead 
III, and in left-sided block the largest deflection ill be downward in lead $I$. The etiological factor of bundle branch or arborization block is more frequently coronary disturbance, but its significance is similar to that of other types of heart biock.

A discussion of Hyocardial disturbances may be divided into preponderance, rnyoardial impaiment, and coronary artery abstruction. If one side of the heart becomes abnomaliy large the direction of the current generated by the muscle contraction will be changed (7), and definite changes will be seen on the electrocardiogram. If the right ventricle is enlarged the main deflection of the $\%, B, S$ group in lead I will be downward, and with enlargement of the Ieft side of the heart. Whe greatest deflection of the $B$, S group will be downward in Iead III. Hore recent investigation (14, 15) has shown that these abnormalities are not always due to enlargement of one side of the heart, but may occur in normal hearts. El ectrocardiographic tracings characteristic of right-sided preponderance may occur in Iong, narrow individuals in whom the apex of the heart is more toward the right; and records similating left-sided preponderance may be produced from short, stocky individuals whose hearts are more transverse 
with in the chest than usual.

Myocardial impaiment per se cannot be diagnosed by the eleatrocardiograph. It is suggested, however, by various abnomalities (I6) of the ventricular complex, such as a widened $\mathrm{a}, \mathrm{h}, \mathrm{s}$ interval, notched or slurred deflections, and various 1 -wave changes, especially a negative T-wave. Low voltage or small deflections of the waves was at one time considered significant of poor muscle function, but this relationship has not proven constant (14, I7). An indirect evidence of myocardial damage is seen in the electrocardiographic records suggesting coronary artery obstruction (18). The $a, B, s$ Group is notohed in at Ieast two leads, left ventricular preponderance is usually shown, the T-wave starts from above the zero Iine and quickly turns away from the starting point without the normal isoelectric period ( the Pardee curve), and the m-wave is usual Iy directed downward in Iead II and one other Iead. The history symptoms and other clinical findings must be considered along with the interpretation of the electrocardiogram. The only congenital condition (14) which gives a constant, significant electrocardiogram is dextrocardia with a change in the relative positions of the heart cavities. 
In this condition the waves in lead $I$ are all pointing in the opposite direction from the normal. They appear as mirrorwriting on the record. If the relationship of the cavities is not disturbed in a dextrocardia the electrocardiogram will be nomal.

The electrocardiogram is not of any appreciable value in the diagnosis of value lesions. It may show preponderance of one of the ventricles, but this evidence is not always reliable, and the $x$ ray or fluoroscope will diagnos enlargement more accurately and more easily (14). From a purely prognostic standpoint it was found by careful tabulation of cases (13) that a negative T-wave in the electrocardiogram is very important. As the close of this paper a few of the figures on negative T-waves will be given. Of 347 patients with mitral stenosis but without negative T-waves $37 \%$ were dead (cardia deaths) within 16 months, while of 43 patients with mitral stenosis, but with negative $\mathrm{T}$-waves $63 \%$ were dead within 12 months. Of 200 patients with rheumatic aortic insufficiency without negative T-waves $39 \%$ were dead wi thin 16 months, while of 62 patients with rheumatio aortic insufficiency plus negative P-waves 
24.

$56 \%$ were dead within 15 months. Of 137 patients with syphilitic aortic insufficiency without negative I-waves $46 \%$ were dead wi thin 16 months, while of 42 patients with syphilitic sortic insufficiency with negative n-waves $76 \%$ were dead in 11 months. 
1. Pardee, H. E. B. - Clinical Aspects of the Electracardiogram Hoeber Inc. New York, 1924 .

2. Garrison, F. H. - His tory of Hedicine, Saunders Co. Philadelphia, 1917.

3. Keating, E. - A.B.G. of the Electrocardiagram, III. I. J. P. 107, ToI LVII, Bebruary, 1930.

4. Hart, T. S. - Reference Han doook of the Medical Solences P. 647; VoI. II, Wood \& Company, 1923.

5. Wiggers, 0. J. - Principles and Practice of Electrocardiography, Losby Company, St. Louis, 1929.

6. Smith, S. A. - Heart Records, Davis Company, Philadelphia 1923.

7. Michie, E. E. - El ectrocardiography. Hili. Surg. P.177P331; Vol. IXVII., Âug. Sept. 1930.

8. BraumeII, C. - Nomal Records, Lancet, P. 409. V01. I, February 1929.

9. Wiep, I. H. - The Electrocardiograph, Its Construction. Principle of Operation, and Clinical Value, U. S. Vet. Bur. Med. Bulletin, P.463, Vol. IV., Hay 1928.

10. Hassee, J. C. - Practical Use of the Blectrocardiograph J. H. A. of teorgia, P. 98, VoI. XIX, 1930.

11. Lewis, J. T. - Olinical Disarders of the Feart Beat. Shaw \& Sons, Chicago, 1925.

12. Thompson, J. C. - EI Ectrocardiagraphic Interpretation, Nebr. State H.J. P.177, VoI. XV, Hay 1930.

13. Willius, F. A. - $\frac{\text { Clinical El ectracardiograms, Daunders co. }}{\text { Philadelphia, 1924. }}$

14. Vaquez, H. - Diseases of the Heart, Saunders Company, PhiladeIphia, 1924. 
15. Iundy, C.J. and Woodruff, I. W. - Experimental Right and Left Axis Deviation, Arch. Int. Hed. P.893, Vol. XIIV., December 1929 .

16. Garr, J. G. - Glinical Significance of the Electrocardiograph, Ned. Clin. of N. H., P.89, VoI. XII, 1928

17. Master, A. W. and Pardee, E. E. B. - Effect of Feart Huscle Disease on the El ectrocardiogram, Arch. Int. Hed. P.42., Vol. XXXVII, 1926.

18. Pardee, B. E. B. - An El ectrocara iographic Sign of Coronary Artery Obstruation, Erch. Int. Ned. P. 244, VoI. XXVI, 1920.

19. Powel1, V. E. - The Electrocardiographic Hethod in Diagnosis, J. U. A. of Georgia, P.100, VoI. XIX, Mheh. 1930.

20. Oppenheimer, B. S. and Bothschild, H. A. - Electrocardiographic Changes Associated with Myocardial Disease. J. h. I. A., P. 429, VoI. IXIX, Mey, 1919.

21. Sigler, L. H. - Lcute Co ronery OccIusion Electrocardiographic study, Ennals of Int. Red. P.969., Vol. IV, February 193I. 